\title{
Diversity Schemes in Interference-Limited Wireless Networks with Low-Cost Radios
}

\author{
Udo Schilcher $^{1}$, Günther Brandner ${ }^{1}$, and Christian Bettstetter ${ }^{1,2}$ \\ ${ }^{1}$ University of Klagenfurt, Institute of Networked and Embedded Systems, Mobile Systems Group, Austria \\ ${ }^{2}$ Lakeside Labs GmbH, Klagenfurt, Austria
}

\begin{abstract}
We investigate the impact of time diversity and space-time diversity schemes on the traffic capacity of large-scale wireless networks with low-cost radios. Especially, we analyze the tradeoff between relaying gain and increased interference due to additional traffic. The results of a simulation-based study imply that cooperative relaying with selection combining shows benefits for low interference scenarios. For high traffic, however, higher-order modulation schemes outperform the investigated cooperative relaying scenario due to lower interference.

Index Terms-Cooperative relaying, interference, network capacity, Rayleigh fading.
\end{abstract}

\section{INTRODUCTION AND Motivation}

Cooperative relaying is a promising technique to mitigate the negative effects of multipath propagation. It can be applied to both relay-enhanced cellular systems and infrastructureless ad hoc networks. Although the past years have seen a tremendous amount of research in this area [1], the majority of work was limited to scenarios with at most four nodes. Questions regarding impact and requirements of cooperative relaying in large networks have not been addressed sufficiently. Our goal is to evaluate cooperative relaying from a networking perspective and address questions such as: What is the impact of cooperative relaying on the overall network performance?

From a physical layer perspective, cooperative relaying can increase the point-to-point throughput between two nodes. If we consider, however, a network of many nodes, these gains do not come for free. From a network perspective, the relay transmissions cause additional interference to adjacent nodes. Assuming low-cost radios, which have neither MIMO nor sophisticated combining techniques, this additional interference reduces the spatial reuse of radio resources in interferencelimited networks.

The goal of this paper is to analyze the network traffic capacity (in terms of subsequent transmissions) if cooperative relaying is used and the node density is high. We compare conventional direct transmission, time-diversity direct transmission, and cooperative relaying by simulations in a Rayleigh block fading channel. In particular, we investigate the tradeoff between exploiting diversity and additional interference caused by this diversity.

The paper is structured as follows: Section II defines the modeling assumptions and explains the transmission methods. Section III contains the setup and the results of the performance evaluation. Next, Section IV covers related work. Finally, Section V concludes.

\section{Modeling Assumptions}

\section{A. Channel Model}

We assume that each node has the same transmission power $p_{t}$ and the same receiver sensitivity $p_{r}^{\min }$. The wireless channel is modeled with a distance-dependent path loss and Rayleigh fading caused by multipath propagation.

For a given receiver, the average power received from a node $i$ located at distance $d_{i}$ from the receiver is given by

$$
\bar{p}_{r, i}=p_{t} g_{\mathrm{ref}} \cdot\left(\frac{d_{i}}{d_{\mathrm{ref}}}\right)^{-\alpha}
$$

with pathloss exponent $\alpha$ and a reference gain $g_{\text {ref }}$ at a reference distance $d_{\text {ref }}$. In the following, we set $d_{\text {ref }}=1 \mathrm{~m}$ and assume $g_{\mathrm{ref}}=1$, such that (1) simplifies to

$$
\bar{p}_{r, i}=p_{t} \cdot d_{i}^{-\alpha}
$$

with the distance $d_{i}$ normalized to meters.

Caused by multipath propagation, several radio waves superimpose vectorially at the receiver. Hence, the scalar amplitude of the signal is Rayleigh distributed. Thus, the instantaneous power received from node $i$ is an exponentially distributed random variable, with probability density function (pdf)

$$
f_{p_{r, i}}(x)=\frac{1}{\bar{p}_{r, i}} \cdot \exp \left(-\frac{x}{\bar{p}_{r, i}}\right) .
$$

We assume that the channel state does not change during a single transmission (block fading) and that the channel coherence time is similar for all scenarios.

\section{B. Node Placement Model}

We consider a network with $n$ nodes randomly distributed on a rectangular area of size $A$. Let $\lambda:=n / A$ denote the density of the nodes. The distance $d$ between two nodes is determined using a wrap-around distance metric to avoid border effects [2].

This paper uses both a homogeneous and an inhomogeneous node distribution. When using a homogeneous node distribution, all nodes are randomly placed in the system area sampling from a uniform distribution.

The inhomogeneous node distribution is generated following the process presented in [3]. It is based on thinning of a homogeneous node distribution. For all nodes the numbers of neighbors within a predefined range $\rho$ are determined, and 
all nodes with less than $k$ neighbors are deleted. To obtain an inhomogeneous distribution with $n$ nodes, the underlying homogeneous distribution must have a significantly higher number of nodes $n_{0} \gg n$. The number of nodes surviving the thinning process depends on the parameters $\rho$ and $k$. It can be estimated by

$$
\mathrm{E}(n)=n_{0}\left(1-\frac{\Gamma\left(k, \rho^{2} \pi n_{0} / A\right)}{(k-1) !}\right),
$$

where $\Gamma(k, \mu)$ denotes the upper incomplete Gamma function.

\section{Traffic Model}

We use the following probabilistic traffic model. Time is divided into periods of equal duration. In each period, $n_{s}$ of the $n$ nodes are selected as senders. Each sender randomly picks an arbitrary node as destination, which must be located within the maximum pathloss range

$$
d_{\max }=\left(\frac{p_{t}}{p_{r}^{\min }}\right)^{\frac{1}{\alpha}}
$$

from the sender. The overall traffic load in the network is thus $n_{s}$ concurrent transmissions per time period.

\section{Interference Model}

Let us now consider the interference at the destination. The node that transmits the desired signal (sender) is indexed by $i=0$. All other nodes transmitting at this time instant cause interference at the destination. We denote the number of interferers with $k$. The instantaneous value of the overall interference $p_{I}$ is a sum

$$
p_{I}=\sum_{i=1}^{k} p_{r, i}
$$

of exponentially distributed random variables $p_{r, i}$. The expected values $\bar{p}_{r, i}$ are in general different, as the distances $d_{i}$ from the interfering nodes are different. Therefore, the sum $p_{I}$ follows a generalized Erlang distribution (for a definition see [4], pp. 41ff), which is a special case of the phase-type $(\mathrm{PH})$ distribution. The corresponding pdf is given by

$$
f_{p_{I}}(x)=-\beta \mathrm{e}^{x \Theta} \Theta \mathbf{1}_{k},
$$

with $\beta=(1,0, \ldots, 0)$ and $\mathbf{1}_{k}=(1, \ldots, 1)$ being vectors of length $k$, and $\Theta$ representing the $(k \times k)$-matrix

$$
\Theta:=\left(\begin{array}{ccccc}
-\frac{1}{\bar{p}_{r, 1}} & \frac{1}{\bar{p}_{r, 1}} & 0 & \cdots & 0 \\
0 & -\frac{1}{\bar{p}_{r, 2}} & \frac{1}{\bar{p}_{r, 2}} & \ldots & 0 \\
\vdots & \ddots & \ddots & \ddots & \vdots \\
0 & \cdots & 0 & -\frac{1}{\bar{p}_{r, k-1}} & \frac{1}{\bar{p}_{r, k}-1} \\
0 & \cdots & 0 & 0 & -\frac{1}{\bar{p}_{r, k}}
\end{array}\right) .
$$

The expected value of this generalized Erlang distribution is

$$
\bar{p}_{I}=\sum_{i=1}^{k} \bar{p}_{r, i}=p_{t} \sum_{i=1}^{k} d_{i}^{-\alpha},
$$

and its variance is given by

$$
\operatorname{var}\left(p_{I}\right)=\sum_{i=1}^{k} \bar{p}_{r, i}^{2} .
$$

The instantaneous signal-to-interference-and-noise ratio (SINR) at the receiver is then given by

$$
\text { SINR }:=\frac{p_{r, 0}}{N_{0} B+p_{I}}
$$

with $N_{0}$ denoting noise power spectral density (in $\mathrm{W} / \mathrm{Hz}$ ) and $B$ denoting the transmission bandwidth (in $\mathrm{Hz}$ ).

\section{E. Transmission Methods}

This paper compares four transmission methods: The first method is direct transmission, in which a source node sends a packet directly to its destination node. The channel coherence time is assumed to be greater than the packet duration. This method serves as reference method. The second method is called double data rate. It is identical to direct transmission, except that it uses twice the data rate, by applying a higherorder modulation scheme. As a consequence, the duration of each packet is halved.

For the third method, the source node is exploiting time diversity by sending each packet twice. For fairness reasons the data rate is doubled. This reduces the interference for each of the two packets by a factor of two due to the halved packet duration. Since, however, each packet is sent twice, the expected interference power caused per transmission is the same as for direct transmission. Due to our assumptions on the channel coherence time, the channel state stays the same for both packets. The interference, however, can change during this time period since in each time slots randomly selected sources start new transmissions while other transmissions end. The transmission succeeds if the destination is able to decode at least one of the two packets correctly.

The fourth method is cooperative relaying. Here, the source sends a packet to its destination. All other nodes in reception range of the source are trying to overhear this packet. If none of them successfully receives the packet, no relaying is done. Otherwise, one of the nodes that has successfully received and decoded the packet is randomly selected to serve as relay for this transmission. This implies that a decode-andforward scheme is used. The random relay selection serves as a bound for cooperative relaying since the performance can be improved by applying more sophisticated relay selection schemes [5]. It forwards the packet to the destination independent of whether the destination already received the message from the source or not. Similar to time diversity, the interference power may be different for both packets received by the destination since the set of active senders may change.

For fairness reasons, the data rate is again doubled in this method. The transmission is successful if at least one packet can be decoded (selection combining). The expected interference power caused by each transmission is the same as for direct transmission. The states for all three channels (source to destination, source to relay, and relay to destination) are stochastically independent due to spatial diversity. 


\section{F. Bit Error Rates}

For the purpose of a fair comparison, we want to use the same amount of transmit energy in all transmission methods. Since time diversity and relaying send two packets for a given transmission, half of the energy should be used for each packet. Thus, the energy per bit has to be halved, which can be achieved by either sending with half transmission power or double bit rate.

In this paper, we use different bit rates. If $b_{r}$ is the bit rate of direct transmission, $2 b_{r}$ is the bit rate of double data rate, time diversity, and cooperative relaying. The different bit rates are implemented using different modulation schemes. For direct transmission, we apply QPSK (quadrature phase shift keying), whose bit error probability is [6]

$$
P_{b}=\frac{1}{2} \operatorname{erfc}(\sqrt{\mathrm{SINR}}) .
$$

For all other methods, we use 16-QAM (quadrature amplitude modulation); its bit error probability can be approximated with [6]

$$
P_{b} \approx \frac{1}{2} \operatorname{erfc}\left(\sqrt{\frac{2}{5} \operatorname{SINR}}\right) .
$$

\section{Performance Analysis}

\section{A. Simulation Setup}

We use four simulation scenarios (see Table I), which differ in the distribution of the nodes and the selection criterion for the destination. The distribution of nodes is explained in Section II-B. The destination is always selected at random from the set of nodes located within the maximum pathloss range of the sender. In scenarios with no criteria, the destination is selected randomly from all nodes in this range, independent of whether or not the node is already receiving or transmitting. In scenarios with only idle nodes, the destination is randomly chosen from the nodes in range that are currently neither transmitting nor receiving.

TABLE I

SIMULATION SCENARIOS

\begin{tabular}{|c||c|c|}
\hline & node distribution & dest. selection \\
\hline \hline Scenario 1 & homogeneous & no criteria \\
\hline Scenario 2 & homogeneous & only idle nodes \\
\hline Scenario 3 & inhomogeneous & no criteria \\
\hline Scenario 4 & inhomogeneous & only idle nodes \\
\hline
\end{tabular}

The simulation parameters are given in Table II. Some parameters must be chosen carefully, to achieve meaningful simulation results:

- The node density is chosen to achieve a good tradeoff between a reasonable number of neighbors per sender and a feasible simulation time.

- The expected interference power at a given time instant can be chosen by defining the number of nodes transmitting simultaneously (only in discrete steps). Hence, the simulation area has to be large enough to allow for a sufficiently high granularity of the simulated interference.
The other parameters have been chosen from state-of-the-art wireless technologies.

TABLE II

SiMULATION PARAMETERS

\begin{tabular}{|l|c|c|}
\hline Parameter & Symbol & Value \\
\hline \hline Number of nodes & $n$ & 2000 \\
\hline Area & $A$ & $30000^{2} \mathrm{~m}^{2}$ \\
\hline Node density & $\lambda=n / A$ & $2.2 \cdot 10^{-6}$ \\
\hline Duration & & 10000 time periods \\
\hline Transmitters per time unit & $n_{s}$ & $1-40$ \\
\hline Transmission power & $p_{t}$ & $0 \mathrm{dBm}$ \\
\hline Receiver sensitivity & $p_{r}^{\min }$ & $-94 \mathrm{dBm}$ \\
\hline Maximum transmission range & $d_{\max }$ & $1360 \mathrm{~m}$ \\
\hline Packet length & & $1024 \mathrm{bit}$ \\
\hline Pathloss exponent & $\alpha$ & 3 \\
\hline Bit rate with QPSK & $b_{r}$ & $250 \mathrm{kbps}$ \\
\hline Noise spectral density & $N_{0}$ & $4.003886 \cdot 10^{-21} \mathrm{~J}$ \\
\hline Bandwidth & $B$ & $2 \mathrm{MHz}$ \\
\hline Thinning range & $r_{0}$ & $1000 \mathrm{~m}$ \\
\hline Thinning limit & $k_{0}$ & 13 \\
\hline
\end{tabular}

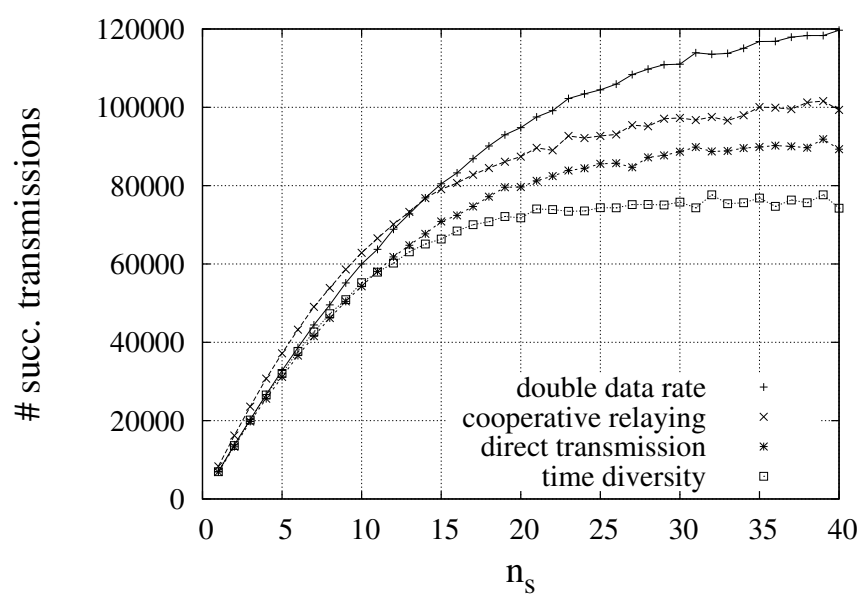

(a) Overall success rates.

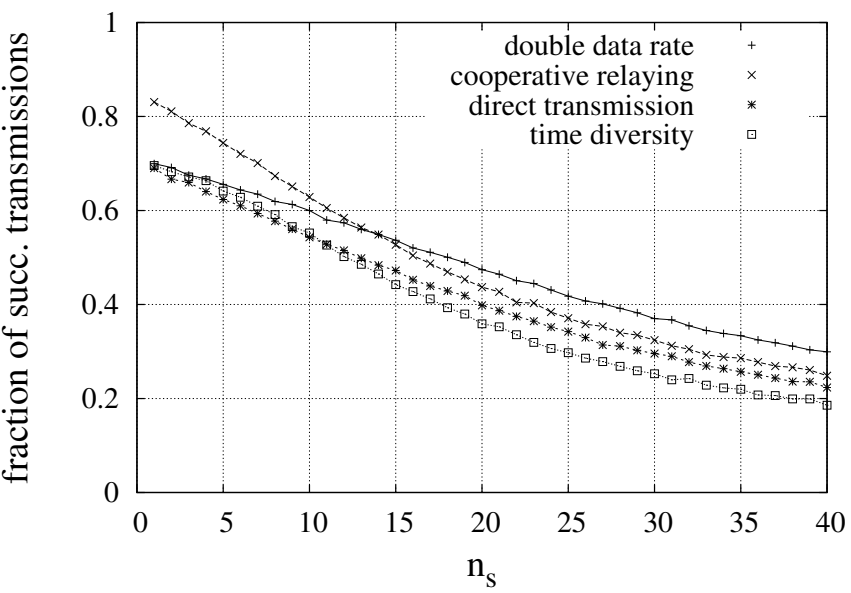

(b) Relative success frequencies.

Fig. 1. Successful transmissions in Scenario 1. 
A simulation run comprises 10000 time periods for each traffic load $\left(n_{s}=1, \ldots, 40\right)$ and each transmission method. In each time period a fixed number $n_{s}$ of senders and corresponding destinations are randomly selected. The duration of a transmission is two time periods for direct transmission and one time period for the other methods. During simulation we collect the following statistics: the overall number of transmissions, the number of successful transmissions, the number of successful packet deliveries for time diversity and cooperative relaying, and the average distances between the nodes.

\section{B. Simulation Results}

The first simulation compares all four transmission methods in Scenario 1. Figure 1 shows (a) the number of successful transmissions and (b) the fraction of successful transmissions as a function of the number of concurrent transmissions $n_{s}$. We can partition the plot into three regimes: the low interference regime $\left(n_{s}=1 \ldots 8\right)$, the medium interference regime $\left(n_{s}=\right.$ $8 \ldots 15)$, and the high interference regime $\left(n_{s}>15\right)$.

In the low interference regime, the main limiting factor for transmission success is the channel state due to Rayleigh fading. Here, cooperative relaying shows the best performance. All other methods are about the same.

As the traffic load increases (medium interference regime), we can observe two major effects: First, double data rate transmission is outperforming both direct transmission and time diversity. The reason for this behavior is that double data rate transmissions generate only half of the interference than all other methods. Therefore, the slope of the curve is reduced, leading to a better performance in high interference situations.

Second, direct transmission is outperforming time diversity, since the interference of two consecutive time slots is highly correlated. Note that this correlation is independent of the channel states that are assumed to be uncorrelated. Hence, if the first transmission fails, the second is also very likely to fail.

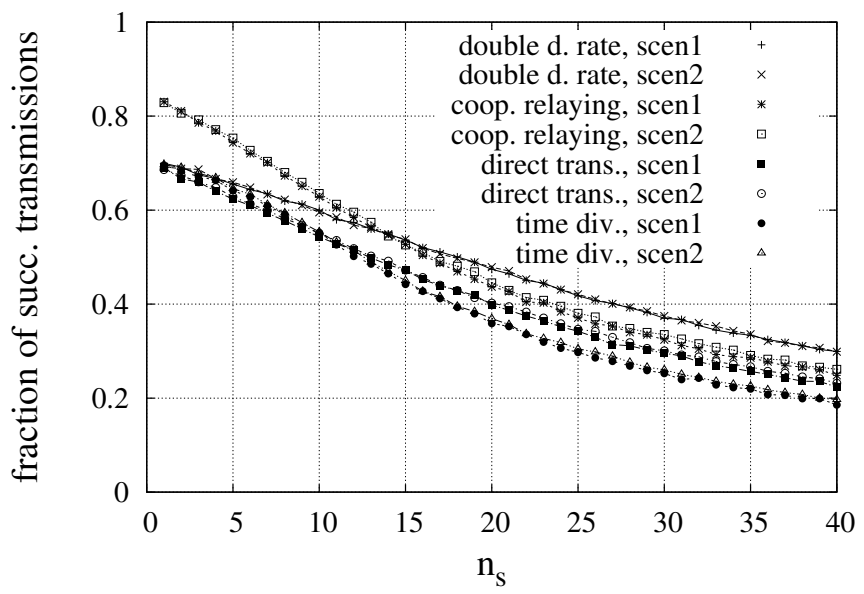

Fig. 2. Relative success frequencies for Scenarios 1 and 2.
In the high interference regime, double data rate transmissions outperform cooperative relaying due to the lower interference. Here, we can speak of a purely interferencelimited scenario. As the traffic load increases much further, the number of successful transmissions does no longer increase but experiences a saturation effect.

For high traffic loads in Scenario 1, the source is likely to select a non-idle node as destination (no criteria). In Scenario 2 only idle nodes are allowed to be destinations. Hence, as can be seen in Figure 2, in the high interference regime we can observe a slight improvement of the performance compared to Scenario 1 . The difference is, however, very small; but it would be significantly higher for a higher number of concurrent transmissions (not shown on the figure). In the low and medium interference regimes there is no difference at all. For double data rate transmissions there can be no difference observed in Figure 2 due to the reduced interference. Again, for a higher number of concurrent transmissions a difference would occur.

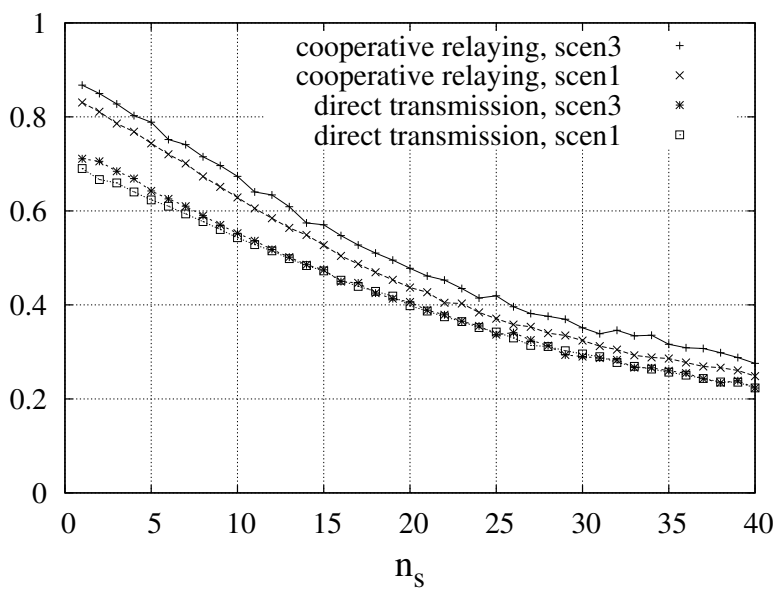

(a) Cooperative relaying vs. direct transmission.

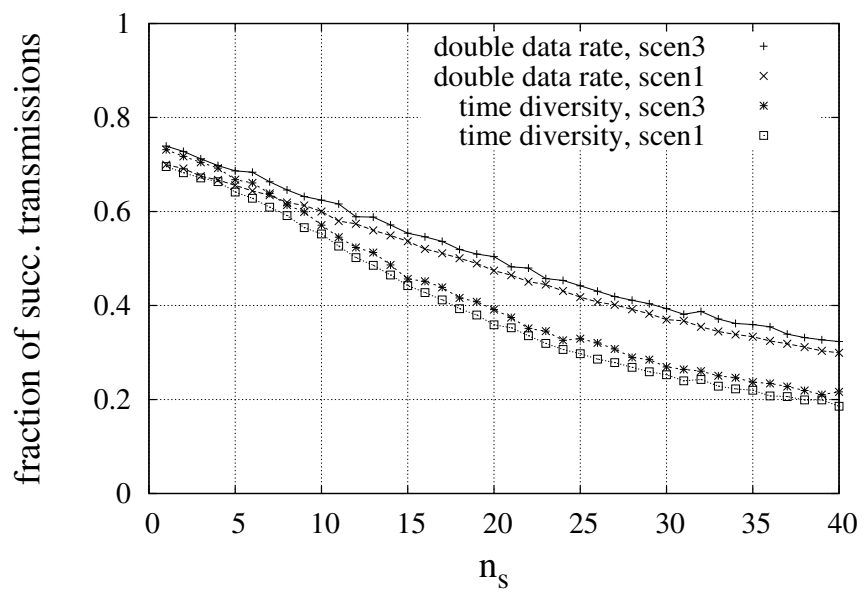

(b) Time diversity vs. double data rate.

Fig. 3. Relative success frequencies for Scenarios 1 and 3. 
When comparing Scenarios 3 and 4 similar effects can be observed; a plot with this comparison is therefore omitted.

Figure 3 compares the transmission methods on homogeneous and inhomogeneously distributed nodes (Scenario 1 vs. Scenario 3). The trends are similar for both homogeneously and inhomogeneously distributed nodes. There is, however, a significant difference: All methods except direct transmission improve their performance for inhomogeneously distributed nodes, which are the methods that use 16-QAM. This effect can be explained by an improved SINR due to a lower average distance between source and destination (and also to the relay nodes).

The lack of improvement for direct transmission is due to the fact that a lower-order modulation scheme has a weaker dependence on a good SINR than a higher-order modulation scheme. Therefore, the advantage of both double data rate and cooperative relaying over direct transmission is higher for inhomogeneously distributed nodes.

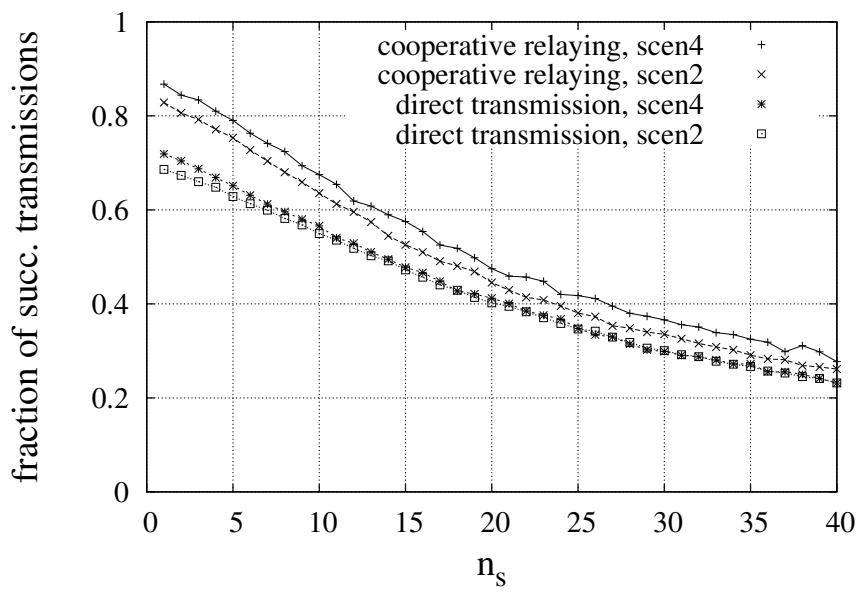

(a) Cooperative relaying vs. direct transmission.

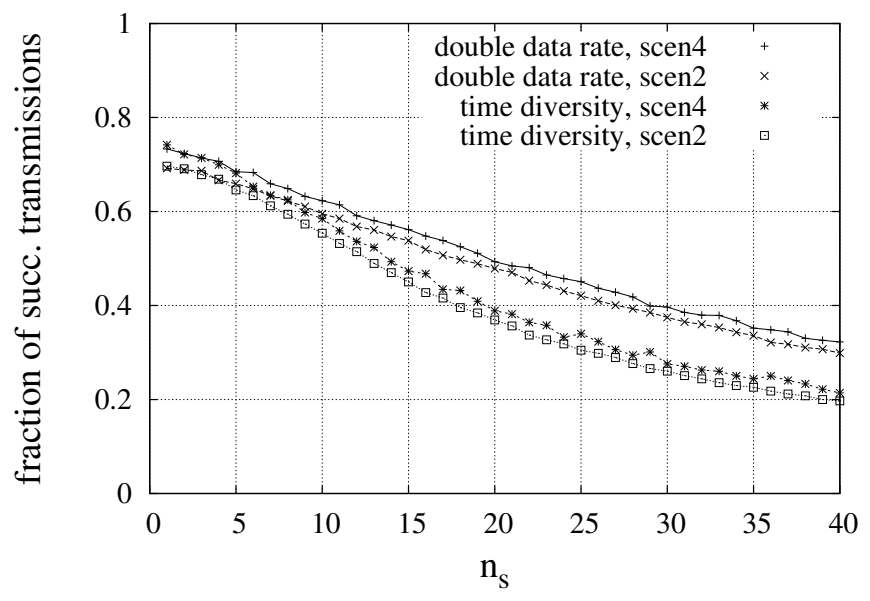

(b) Time diversity vs. double data rate.

Fig. 4. Relative success frequencies for Scenarios 2 and 4.
In Scenario 4 an increase of the performance in the high interference regime compared to Scenario 3 can be observed. This difference is again due to non-idle destination nodes in this scenario. Overall, Scenario 4 leads to the highest network capacity.

As shown in Figure 4, an inhomogeneous node distribution shows an advantage especially for cooperative relaying, independent of the traffic load. A similar behavior can also be observed for double data rate and time diversity (shown in Figure 4(b)). However, direct transmission performs similar for homogeneous and inhomogeneous node distributions. The reasons for this behavior are the same as described in the comparison of Scenarios 1 and 3.

\section{RELATED WORK}

The authors of [7] simulate the performance of cooperative diversity strategies in interference-limited ad hoc networks. They conclude that the gain of cooperative diversity depends heavily on the chosen cooperative strategy and on the resource allocation strategy. Contrary to our work, the nodes are distributed on a grid in a deterministic manner and each node can only transmit to the surrounding 8 nodes.

The paper [8] simulates the throughput performance of a 10-user ad hoc network employing cooperative diversity techniques; 20 random topologies are regarded. The average throughput for different system loads with cooperative relaying is obtained and compared to the IEEE 802.11 standard without relaying.

Paper [9] analyzes the capacity of wireless networks under the relay traffic pattern. In the underlying model there is only one source-destination pair present, while the other nodes act as relays during the transmission. The authors derive upper and lower bounds for the network capacity and show that these bounds are equal when the number of nodes approaches infinity.

The performance of three different relaying strategies are investigated in [10]. The results are based on the study of the transmissions of one source-destination pair, where either one or two relay nodes are placed in between. These results, however, do not consider the effects of interference caused by concurrent transmissions of other nodes in the network.

The authors of [11] propose and study a receiver-centric model of interference, where each node possibly disturbs the transmission of other nodes that are located within a defined interference radius. The degree of interference at a certain receiver depends on the number of interference radii that cover this receiver. In contrast to the paper at hand there is a strict border that determines if a sender interferes with other nodes.

For theoretical approaches for modeling interference, the following papers can be considered: Article [12] derives analytical expressions for different measures of the network throughput in wireless networks. It classifies the networks in an uncertainty cube regarding node placement, fading, and MAC protocols and derives analytical results. A comprehensive overview of these results can also be found in [13]. In contrast to our work transmission success is determined based 
on a threshold value for SINR. Further, we contribute by also considering inhomogeneous node distributions.

In [14] the author derives the probability density functions for the SINR and the bit error probability for Rayleigh fading channels. An additive interference model and a capture threshold model for random access networks and scheduled networks are discussed in [15].

The paper [16] proposes an interference model for microcellular networks, which incorporates user terminal mobility and radio propagation parameters.

In [17] the authors present a circle-based interference model. The circles are chosen based on the magnitude of interference caused by a node located within it. Using these circles, the authors estimate the transmission success probability. In [18] also a multiple circle model is introduced. The authors use it to compute transmission success probability and apply the model to a MAC protocol. The major difference to [17] is the hard cutoff for the SINR value instead of a mapping from SINR to bit error probability.

\section{CONCLUSions AND Further WORK}

In the paper at hand a simulation-based study of direct transmission, double data rate transmission, time diversity, and cooperative relaying in interference-limited dense networks is presented. The comparisons are conducted on scenarios that differ in the node distribution (homogeneous vs. inhomogeneous distribution) and the criterion for selecting the destination (all nodes vs. only idle nodes).

Under the assumptions given, the main results can be summarized as follows: Cooperative relaying methods are performing well in all scenarios, especially better than direct communication. In interference-limited networks it is, however, advantageous to use higher-order modulation schemes or even adaptive modulation instead of cooperative relaying if no combining techniques are used. The improvement of the SINR due to the reduced interference is outbalancing the diversity gain of cooperative relaying. When applying both cooperative relaying and high modulation schemes, the higher interference of relaying is decreasing the performance significantly.

Regarding the criterion for selecting the destination nodes, a slight performance improvement can be achieved by selecting only idle nodes as destinations. For real protocols, however, the signaling overhead for identifying idle nodes as potential destinations has to be considered. Here, a more detailed analysis has to be performed to determine whether applying such a criterion is still advantageous.

Our next steps comprise a deeper analysis of more sophisticated cooperative relaying (on demand relaying) and time diversity algorithms that send the second packet only if necessary. This, on the one hand, minimizes the interference but, on the other hand, introduces additional signaling overhead. Besides that, we will perform an analytical investigation of the success probabilities for different transmission methods, based on the statistical channel model presented in this paper.

\section{ACKNOWLEDGMENTS}

This work was performed in the project Cooperative Relaying in Wireless Networks (RELAY) of the research cluster Lakeside Labs and was partly funded by the European Regional Development Fund, the Carinthian Economic Promotion Fund (KWF), and the state of Austria under grant 20214/15935/23108.

\section{REFERENCES}

[1] K. J. R. Liu, A. K. Sadek, W. Su, and A. Kwasinski, Cooperative Communications and Networking. Cambridge University Press, 2009.

[2] C. Bettstetter and O. Krause, "On border effects in modeling and simulation of wireless ad hoc networks," in Proc. IEEE Intern. Conf. on Mobile and Wireless Commun. Networks (MWCN), Aug. 2001.

[3] C. Bettstetter, M. Gyarmati, and U. Schilcher, "An inhomogeneous spatial node distribution and its stochastic properties," in Proc. ACM/IEEE Intern. Symp. on Modeling, Analysis, and Simulation of Wireless and Mobile Systems (MSWiM), 2007.

[4] M. F. Neuts, Matrix-Geometric Solutions in Stochastic Models. The John Hopkins University Press, 1981.

[5] H. Adam, C. Bettstetter, and S. M. Senouci, "Adaptive relay selection in cooperative wireless networks," in Proc. IEEE Intern. Symp. on Personal, Indoor and Mobile Radio Communications (PIMRC), Sept. 2008.

[6] A. Goldsmith, Wireless Communications. Cambridge University Press, 2005.

[7] H. Zheng, Y. Zhu, C. Shen, and X. Wang, "On the effectiveness of cooperative diversity in ad hoc networks: a mac layer study," in Proc. IEEE International Conference on Acoustics, Speech, and Signal Processing (ICASSP), vol. 3, pp. 509-512, Mar. 2005.

[8] M. Islam and W. Hamouda, "Performance of cooperative ad-hoc networks in rayleigh fading channels," in Proc. IEEE Vehicular Technology Conference (VTC), pp. 1-4, Sept. 2006.

[9] M. Gastpar and M. Vetterli, "On the capacity of wireless networks: The relay case," in Proc. IEEE INFOCOM, pp. 1577-1586, 2002.

[10] S. Gormus, D. Kaleshi, J. McGeehan, and A. Munro, "Performance comparison of cooperative and non-cooperative relaying mechanisms in wireless networks," in Proc. IEEE Wireless Communications and Networking Conference, vol. 2, pp. 890 -896, Apr. 2006.

[11] P. Rickenbach, S. Schmid, R.-Wattenhofer, and A. Zollinger, "A robust interference model for wireless ad-hoc networks," in Proc. IEEE International Parallel and Distributed Processing Symposium, pp. 66-80, Apr. 2005.

[12] M. Haenggi, "Outage, local throughput, and capacity of random wireless networks," IEEE Trans. Commun., vol. 8, Aug. 2009.

[13] M. Haenggi and R. Ganti, Interference in Large Wireless Networks. now publishing, 2009

[14] K. Hamdi, "Exact probability of error of bpsk communication links subjected to asynchronous interference in rayleigh fading environment," IEEE Trans. Commun., vol. 50, pp. 1577-1579, Oct 2002.

[15] A. Iyer, C. Rosenberg, and A. Karnik, "What is the right model for wireless channel interference?," IEEE Trans. Wireless Commun., vol. 8, pp. 2662-2671, 2009.

[16] B. C. Jones and D. J. Skellern, "An integrated propagation-mobility interference model for microcell network coverage prediction," Wireless Personal Communications, vol. 5, pp. 223-256, 1997.

[17] U. Schilcher, M. Gyarmati, G. Brandner, and C. Bettstetter, "Modeling interference in wireless networks," Poster presentation at ACM MobiCom, Sept. 2009.

[18] G. Parissidis, M. Karaliopoulos, M. May, T. Spyropoulos, and B. Plattner, "Interference in wireless multihop networks: A model and its experimental evaluation," in Proc. WoWMoM, pp. 1-12, June 2008. 